\title{
Uso de berramientas digitales multimedia en la enseñanza- aprendizaje de la asignatura de Embriología Humana en estudiantes de la Carrera de Medicina de UNAN-Managua
}

MSc. Moisés Acevedo Flores

Médico y Cirujano

UNAN-MANAGUA, Facultad de Ciencias Médicas moisesacevedo@yahoo.com

Palabras clave: Embriología, Tecnología de Información y Comunicación (TIC), Estudio Descriptivo, Correlacional y Analítico.

\section{RESUMEN}

E I tema del uso de la tecnología en la educación es de constante discusión con resultados muy variados, sea de mucho o de poco éxito según variadas condiciones. Con el propósito de evaluar los resultados del uso de herramientas digitales multimedia en el aprendizaje de la Embriología Humana, se realizó un estudio de tipo observacional, descriptivo, prospectivo, transversal, analítico con enfoque filosófico mixto. Realizado con 75 estudiantes del segundo año de Medicina de la UNAN-Managua, durante el primer semestre del 2016. No hubo significancia estadística entre tres modalidades de estudio; la primera, sin el uso de herramientas multimedia; y otras dos, utilizando ayudas con y sin supervisión respectivamente. La herramienta más utilizada y aceptada fue videos cortos. No todos los estudiantes poseen equipos para reproducir archivos digitales, el teléfono celular fue el de posesión más común, no hubo significancia estadística entre los tipos de dispositivos electrónicos usados por los estudiantes y los resultados de las notas finales. El uso de instrumentos cualitativos permitió formular un plan de acción para optimizar el uso de herramientas digitales en la asignatura de Embriología Humana.

\section{INTRODUCCIÓN}

El aprendizaje, el estudio y la educación juegan un papel relevante en el desarrollo de la sociedad, siendo uno de los motores de su proceso evolutivo. Por esa razón, la docencia y los procesos de enseñanza-aprendizaje deben adaptarse permanentemente a las características de los individuos que en cada momento la componen. Por otro lado, se está viviendo en los últimos años una auténtica revolución tecnológica que cambia los hábitos de vida y afecta el entorno, en ocasiones, saturado o desbordado por tanta información.

Los primeros indicios de investigación sobre el uso de las TIC en la educación se encuentran alrededor de 1918, pero se considera la década de los 50 como el punto clave de desarrollo significativo. La década de los 70 aporta el despegue de los medios de comunicación en masa como una gran influencia social; a partir de esta época el desarrollo de la informática consolida el uso de los ordenadores para fines educativos, concretamente en aplicaciones conocidas como 
Enseñanza Asistida por Ordenadores (EAO). Con la aparición de los ordenadores personales esta opción se generaliza como alternativa de enormes posibilidades (Vidal, 2006).

Una de las primeras propuestas significativas es la formulada por Salomón y Clark (2007) encaminada a encontrar el medio más eficaz y útil para el proceso enseñanza-aprendizaje, evaluando, en un segundo lugar, a quien se le entrega el material, cómo se le entrega y qué se entrega.

El presente informe aborda la evaluación del uso de herramientas digitales multimedia y su influencia en el proceso enseñanza-aprendizaje en un grupo de estudiantes del segundo año de la carrera de Medicina en la Facultad de Ciencias Médicas del Recinto Universitario Rubén Darío.

En este estudio se utilizó herramientas digitales sencillas y amigables asistidas por Tecnología, como un medio para ser implementadas y evaluadas en estudiantes de la Facultad de Ciencias Médicas en su aceptación, utilidad y aporte para mejorar el aprendizaje y que sirva como punto de partida de muchos esfuerzos futuros.

\section{METODOLOGÍA}

Se realizó una investigación observacional, descriptiva, en la que se correlacionaron distintos factores con resultados posteriores a una prueba escrita realizada a los estudiantes con base a un tema de Embriología Humana y se planificaron distintos escenarios de aprendizaje donde se involucró el uso de herramientas digitales multimedia. Se utilizaron instrumentos, métodos y técnicas cualitativas y cuantitativas, por tanto, se implementó un enfoque mixto.

El estudio se dividió en tres momentos: el primero, que consistió en el diseño de las herramientas digitales multimedia; el segundo, cuando se procedió al encuentro con los estudiantes para aplicarles la correspondiente modalidad de estudio y su respectiva evaluación; y un tercer momento, en el que se aplicaron los instrumentos de recolección de la información cualitativa, como fueron los grupos focales y entrevistas individuales.

Para elaborar las herramientas virtuales se realizó una planificación consensuada de los contenidos con la ayuda del colectivo docente de la asignatura de Embriología Humana. La planificación digital se hizo con la colaboración de un diseñador gráfico. Se elaboró una aplicación multimedia interactiva utilizando la Suite Adobe CS5. Al resultado del tratamiento y manipulación de todo el material de la multimedia interactiva se le dio salida en un formato "exe", como una aplicación capaz de ser leída en ambiente Windows en cualquier versión superior a XP. Además, se grabó una conferencia explicativa con diapositivas de Power Point y la voz del docente en Camtasia Estudio 7, con una duración de 29 minutos y se le dio salida en formato MP4. Luego se digitalizó el capítulo 3 del libro "Embriología de Langman en su onceava edición": Primera semana del desarrollo de la ovulación a la implantación. El formato de salida de este documento fue en PDF.

En el segundo momento, se escogieron tres grupos de estudiantes de 25 integrantes cada uno y se les asignaron las modalidades de estudio a seguir. Se procedió de la siguiente manera: al grupo 1 de estudio se le designó como el "control". Se convocó para una participación voluntaria y se les citó para recibir la conferencia "Primera semana de desarrollo", la que se hizo de la forma tradicional, con el uso de una presentación en Power Point con una duración de 90 minutos. De inmediato se les facilitó una copia en físico del capítulo 3 del libro "Embriología de Langman en 
su onceava edición": Primera semana del desarrollo de la ovulación a la implantación. Al grupo 2 de estudio se le convocó voluntariamente para su participación, se le informó que a través de sus correos electrónicos se le haría llegar todo el material digitalizado que incluía: un video con la narración del docente de una conferencia acortada en Power Point con duración de 29 minutos, la conferencia original en Power Point en su formato nativo PPT, la copia del capítulo del libro en formato PDF y la aplicación multimedia diseñada para tal fin. Se les orientó cómo utilizar el material de manera breve y además se les envió instrucciones escritas en formato PDF. Al grupo de estudio 3 se le convocó voluntariamente y se le citó para un encuentro supervisado en los laboratorios de informática de la facultad de Ciencias Médicas. El día de la sesión se habilitó 25 computadoras y se les cargó la aplicación multimedia interactiva diseñada para tal fin. Los estudiantes interactuaron con la aplicación durante 90 minutos. Un docente de la asignatura estuvo presente para aclarar cualquier duda del contenido de la aplicación, así como de su uso. Al finalizar el encuentro, se descargó en sus memorias personales todo el material digital disponible ya mencionado, incluyendo la aplicación que utilizaron.

Para todos los grupos se les citó cuatro días posteriores a su encuentro con el docente para ser evaluados mediante prueba escrita con un valor de 100 puntos, sesenta del contenido teórico y 40 del contenido práctico (dibujos, imágenes y nombre de estructuras).

\section{RESULTADOS Y DISCUSIÓN}

La población estudiada estaba entre los 16 y 24 años de edad. La procedencia fue en su mayoría urbana, con un porcentaje de 80 \%. El sexo femenino prevaleció con un 65 \% de participación.

Para establecer relación entre el nivel de aprendizaje de estudiantes que utilizan solamente el método tradicional y los que usan herramientas digitales multimedia como apoyo en el estudio, se realizó un ANOVA de un solo factor, siendo este, la nota obtenida en la evaluación. Se evidencia que ninguna modalidad de estudio fue superior a la otra $(\boldsymbol{p}>\mathbf{0 . 0 5})$. Los contrastes ortogonales realizados para buscar superioridad específica de alguno de los tratamientos tampoco fueron significativos. El promedio de notas para los tres grupos de estudio fue muy similar y cercano a los 60, en una escala de 100 puntos de la prueba escrita. Se observó notas sobresalientes y reprobadas en los tres grupos (máxima 96, mínima 28). Esta falta de significancia es encontrada en otros estudios consultados como el informe de la comisión Europea en 2004 sobre los nuevos entornos de aprendizaje, quien concluye que los nuevos entornos de aprendizaje no dependen tanto del uso de las TIC en sí, sino más bien de la reorganización de la situación de aprendizaje y de la capacidad del profesor para utilizar la tecnología como soporte de los objetivos orientados a transformar las actividades de enseñanza tradicional. De igual forma ocurre en el estudio exploratorio descriptivo que analiza el uso de la multimedia interactiva, como recurso pedagógico en Semiología y su aplicabilidad en la enseñanza de la enfermería (Santiago \& Shiratori, 2009), quienes concluyen que la multimedia es muy útil, sin embargo, no suficiente por sí sola; además, destaca la importancia de la actualización e involucramiento de los docentes para lograr mejores resultados

En este sentido, la información brindada por los instrumentos cualitativos parece orientar sobre los factores que influyeron en este hecho, como falta de incentivo en formar un puntaje real y útil para cursar la asignatura, falta de tiempo y dedicación, falta de planificación en la jornada de estudio. Esto inclina a pensar que es relevante que la responsabilidad de cada individuo sobre 
el compromiso adquirido de estudiar a conciencia para rendir en una prueba posterior sin que hubiese una nota válida de por medio. Las herramientas son pues, eso, auxiliares para hacer más o menos pesada la jornada de aprendizaje.

Ya la teoría nos aclara, que el uso de herramientas pertenecientes a nuevas tecnologías en el proceso educativo es altamente complejo y no concluyente, pero que marca pautas muy claras como la intervención oportuna del docente, actualización e involucramiento del mismo, así como una evaluación constante para encontrar debilidades y potencializar fortalezas. Otros estudios, como el que realizó Torales Chaparro en España, contaron con una estructura completa y organizada y fue inserto en el curso de Radiología con un puntaje válido para aprobar dicha asignatura, en un espacio virtual de estudio y múltiples encuentros con los profesores. Aún con todas estas ventajas organizativas, el estudio no fue concluyente en cuanto a la mejora del rendimiento, utilizando medios digitales, sin embargo, sí logró un mayor índice de estudiantes que se presentaron a realizar sus pruebas evaluativas que aquellos con clases solamente presenciales, siendo mucho menos los desertores del curso en la modalidad virtual.

Si bien los contrastes ortogonales coinciden con los resultados del ANOVA, en el hecho de ser estadísticamente no significativos, los resultados de las medias comparadas que muestran una ligera variación a favor de los estudiantes que tuvieron una sesión controlada y supervisada con la multimedia interactiva, permite sugerir que para estudios futuros se delimite mejor y de forma más consistente, el control de los tratamientos, lo que permitiría evaluar más objetivamente el desempeño de las herramientas multimedia.

La utilidad en todos sus aspectos resultó con un elevado porcentaje entre las categorías de muy útil y útil, ninguno de los encuestados la consideró como inútil. Al utilizar la prueba de asociación Gamma en las variables utilidad para optimizar tiempo y utilidad para comprender contenidos teóricos resulta una relación significativa, lo que puede interpretarse como que aquellos estudiantes que percibieron usar mejor el tiempo, también percibieron comprender mejor los contenidos teóricos del tema. Este resultado apunta más a los aspectos individuales del manejo del tiempo y organización que a las herramientas en sí. Esto es coincidente con Ávila y Samar (2002) quienes concluyen que las nuevas tecnologías de información y comunicación permiten al estudiante ser el principal actor en la construcción de sus conocimientos con base a situaciones diseñadas por el docente.

La percepción de los estudiantes, en cuanto al papel de los medios digitales, parece estar clara, no sustituyen nada, pero sí complementa el estudio y hubo consenso en la necesidad de implementar estas herramientas para mejorar el aprendizaje en toda la carrera. Esto coincide con la conclusión casi unánime de toda la teoría consultada, el catedrático es indispensable y los textos, un medio para llegar al conocimiento profundo. Inclusive, la utilización inadecuada de estas tecnologías puede resultar infructuosa, pues la propuesta ha de ser científicamente sustentada y con una base didáctica clara y definida, de lo contrario, se disminuirán las ventajas de estos entornos (Herrera, 2004).

El uso y la utilidad de la aplicación multimedia se discutirá por separado en esos dos aspectos: respecto al uso, destaca el hecho que solamente la mitad la utilizó y que la mayoría de esa mitad estuvo en la sesión supervisada por el docente. Al parecer, este acompañamiento generó cierto interés en usarla como herramienta. La significancia estadística para esta relación fue concluyente. 
Una vez más queda claro que la presencia y motivación del docente es un factor determinante en la actitud del estudiante frente al material asignado; no basta con solo entregar el material, es necesaria la motivación que se genera para sugerir su utilidad. Aquellos que solo recibieron la aplicación por correo electrónico tuvieron una tendencia a desestimarla como herramienta de estudio.

Respecto a la utilidad, se presentaron dificultades que no permiten hacer un análisis completo de la aplicación. En principio, las dificultades técnicas y de recursos, como un audio inadecuado en los videos cortos, poca calidad de la imagen en ciertos videos y el exceso de contenido teórico en algunas secciones, hicieron de esta herramienta poco provechosa. Otra dificultad fue la accesibilidad, el formato de salida fue para lectura en sistemas operativos de computadoras y no se contempló su uso para sistemas Android, propio de Celulares y Tabletas, esto redujo considerablemente su oportunidad de uso ya que solamente un 61 \% de los estudiantes tenían disponible una laptop y solo un 40 \% tenía una computadora de escritorio en su hogar. Otro aspecto que analizar de la multimedia fue el contexto de su uso, esta herramienta se envió como un archivo más y la forma apropiada debió ser como parte integral de una plataforma educativa, es decir, un entorno virtual estructurado o en su defecto como App o aplicación para sistemas Android, como se comentó anteriormente. El tener la herramienta aislada, sumado a las dificultades técnicas expresadas por los estudiantes, al parecer redujo su uso y por ende, su utilidad, debido a que este estudio no contempló el diseño de un entorno virtual completo en Moodle u otra plataforma.

El uso de un video corto de 29 minutos, en el cual un docente explica las diapositivas a manera de conferencia virtual, constituyó la herramienta con mayor uso y preferencia. Para los 50 estudiantes que recibieron material digital, este video fue el único vínculo entre su aprendizaje y el docente en cuanto a compartir el tema a evaluar. No constituyó un sustituto de la conferencia ya que su duración fue acortada, por ende, el número de diapositivas y careció de la posibilidad de responder a preguntas o dudas. Pese a eso, fue muy apreciado como herramienta introductoria para enfrentar el texto del libro con mayores elementos y comprensión. El video cumple con todas las características de ser multimedia, ya que involucra sonido e imagen, cumpliendo con los dos canales para procesar la información sugeridos por Mayer. Esta forma de material tuvo la ventaja de la flexibilidad de tiempo y repetición a voluntad. Todas las ventajas fueron referidas y reforzadas en las entrevistas y grupos focales a los estudiantes.

El capítulo en digital del libro Guía en formato PDF fue apreciado por su utilidad práctica. Al no llevar los estudiantes la asignatura de Embriología en este semestre, carecían del texto en físico. Sin embargo, siempre se observa que muchos de ellos no tienen la opción de obtener el libro aún llegado el momento, por lo que al parecer, esto podría ser una experiencia a considerar en el futuro. Esto podría conllevar conflictos de derecho de autor por lo que habría que considerar este aspecto antes de implementarlo como práctica oficial en la Facultad de Ciencias Médicas.

En una generación net, era de esperar que la totalidad de estudiantes contaran con dispositivos de gran conectividad digital, sin embargo, diferentes razones pudieron estar involucradas para que esto no fuese posible. En general, la posesión de dispositivos no sobrepasó el 63 \% en el más accesible de los aparatos, el teléfono inteligente; casi un $10 \%$ de los participantes no disponen de ningún aparato reproductor de información digital. Este estudio no profundiza en las causas o 
razones para este fenómeno, pero es un dato a considerar, sobre todo, cuando hay una tendencia a dar por sentado que "todos" los universitarios están conectados mediante la tecnología.

\section{CONCLUSIONES}

1. No hubo relación estadística en el nivel de aprendizaje, medido mediante calificación de una prueba escrita, entre los estudiantes que utilizan solamente el método tradicional y los que usan herramientas digitales multimedia como apoyo en el estudio (capítulo en PDF, video corto de la conferencia, multimedia interactiva y diapositivas de la conferencia en Power Point) lo que indicó un rol relevante del individuo y sus prioridades de estudio, así como la organización del tiempo por sobre el material recibido, digital o no.

2. La utilidad del uso de las herramientas digitales multimedia en el aprendizaje de la Embriología Humana, estuvo en su mayoría en la categoría de muy útil a útil en la escala Likert. Hubo relación estadística entre la optimización del tiempo y la comprensión de los contenidos teóricos. Fue casi un consenso, que las herramientas son complementarias y no sustitutivas. La totalidad de los participantes consideran que es pertinente utilizar estos medios como auxiliares de estudio en la carrera. La herramienta digital con mejor calificación fue el video corto de la conferencia.

3. No todos los estudiantes poseen dispositivos electrónicos para correr archivos digitales. El celular inteligente fue el más común en posesión. No se encontró correlación estadística entre el nivel de aprendizaje de la Embriología Humana y la posesión de dispositivos electrónicos.

4. El uso de métodos y técnicas mixtas permitió obtener suficiente información para proponer un plan de implementación de algunas herramientas digitales con gran aceptación en las distintas modalidades de estudio.

\section{REFERENCIAS BIBLIOGRÁFICAS}

Akyeampong, K. (2009). Las tecnologías multimedia y reforma educativa en Africa: el caso de Ghana. Comunicar, $n^{\circ} 32$, v. XVI, 2009. Revista Científica de Educomunicación, 109118.

Ávila, R. E., Samar, M. \& Diego, C. (2002). El uso de las nuevas tecnologias de informaciòn y comunicaciòn (NTIC) para la enseñanza/ aprendizaje de la Biologia celular, Histologia y Embriología. Il congreso Iberoamericano de Informática médica, Nov 4 a Nov 30. disponible en: http://www.informedicajournal. org/a1n2/files/papers_informedica/avila1.pdf

European Comission. (2004). Study of Innovative learning environmentes in school edutation. Final report.ICT@Europe.edu.
Fandos, M. \& Jiménez, J. (2002). Estrategias didácticas en el uso de las tecnologías de la información y la comunicación. Acción pedagógica, 28-39.

Fernández, R. R., Server, P. M. \& Carballo, C. E. (2006). Aprendizaje con nuevas tecnologías paradigma emergente. ¿Nuevas modalidades de aprendizaje? EDUTEC. Revista Electrónica de Tecnología Educativa, 20, 1-24. Recuperado de: http://edutec.rediris.es/ Revelec2/revelec20/raul20.pdf

Ferreiro, R. (2012). Cómo ser mejor maestro. El método ELI. México: Trillas.

Ferreiro, R. \& Margarita, E. (2012). El ABC del aprendizaje cooperativo. México: Trillas. 
García, V. M. \& Cabrero, J. (2007). Uso del Multimedia Interactivo en el contexto universitario. Pixel Bit. Revista de medios y comunicación, vol.30. Disponible en: http:// www.redalyc.org/articulo.oa?id=36803002), 17-30

González, L. \& Tercero, M. (2014). Aplicacion Web con énfasis multimedia para alumnos de segundo año de enfermería en procedimientos básicos de enfermería de la FAREM-Matagalpa durante el año 2013. Matagalpa: UNAN-Managua. Disponible en: http://www.biblioteca.unan.edu.ni:9090/ bases/bcurm/www/4620/index.html

Hamui, A. \& Lavalle, C. (2013). Uso de la tecnología con fines educativos en residentes $y$ profesores de especialidades médicas. Revista Mexicana de Medicina Interna, 558570.

Hernández Sampieri, R., Fernández Collado, C. \& Baptista Lucio, P. (2010). Metodología de la investigación. México: Mc Graw Hill.

Herrera, M. A. (2004). Las nuevas tecnologías en el aprendizaje constructivo. OEl. Revista Interamericana de Educación, 34(4), 1-19. Disponible en: http://www.redkipus.org/aad/ images/recursos/33-NT_Aprendizaje_2004. pdf.

Latapie Venegas, I. (2007). Acercamiento al aprendizaje multimedia. Investigación universitaria multidisciplinaria, 6(6), 7-14. Disponible en: file:///C:/Users/pc/Downloads/ Dialnet-AcercamientoAIAprendizajeMultime dia-2695335.pdf.

Lopategui, E. (s.f.). Historia de las computadores. Recuperado el 27 de enero de 2015, de http://www.saludmed.com/EGIC1000/pdf/ Ciencias_de_la_Computadora.pdf

Piura, J. (2012). Metodología de la investigación científica: Un efoque integrador (7a. ed.). Managua.
Ramirez, L. V. (2008). El nuevo paradigma de la Educaciòn a distancia. Algunos criterios de calidad para el porvenir. Revista de Geografía Agrícola, 73-89. Disponible en: http://www. redalyc.org/articulo.oa?id=75711534007.

Ramirez, V. L. (2008). El nuevo paradigma de la educación superior a distancia. Algunos criterios de calidad para el porvenir. Revista de Geografía agrícola, 73-89. Disponible en: $\quad$ http://www.redalyc.org/articulo. oa?id=75711534007.

Ramos, M. B. \& Carlos, V. (2008). El uso de la tecnología multimedia para la enseñanza de la historia de la medicina. Estudio de casos y controles en estudiantes de Medicina. Medigraphic. Artemisa en línea. Revista de la Facultd de Medicina de la UNAM, 252-256.

Salomon, R. \& Clark, R. (2007). Reexamining the mehtodolgy of research on media technology in education. Review of Educational Resaerch, 99-120.

Santiago, L. \& Shiratori, K. (2009). Multimedia interactiva como recurso de enseñanza de semiología en enfermería. Enfermería Global (16), 1-12. Disponible en: http://revistas.um.es/ index.php/eglobal/article/view/66231.

Schalk Quintanar, A. E. (2010). El Impacto de las TIC en la educación. Brasilia: UNESCO.

Torales Chaparro, O. (2008). Diseño y Evaluación de una Aplicación Multimedia para la Enseñanza de Radiología a Alumnos de Medicina (AMERAM). Málaga. : Tesis doctoral. Universidad de Málaga. Disponible en: http:// www-rayos.medicina.uma.es/Rmf/Tesis/ Tesis_Doctoral_Oscar_Torales.pdf.

UNAN-Managua. (2011). Modelo Educativo, Normativa y Metodología para la Planificaión curricular 2011. Managua: UNAN-Managua.

Vidal, María del Pilar. (2006). Investigación de las TIC en la educación. Revista Latinoamericana de Tecnología Educativa, 539-552. 